\title{
Robust Exponential Attractors for Singularly Perturbed Phase-Field Equations with Dynamic Boundary Conditions
}

\author{
Ciprian G. Gal, Maurizio Grasselli and Alain Miranville
}

\begin{abstract}
We consider a singularly perturbed phase-field model of Caginalp type which is thermally isolated and whose order parameter $\phi$ is subject to a dynamic boundary condition. More precisely, we indicate by $\varepsilon$ a (small) coefficient multiplying $\partial_{t} u$ in the heat equation, $u$ being the temperature, and we construct a family of exponential attractors which is robust as $\varepsilon$ goes to 0 . This is physically meaningful since the limiting problem is the viscous Cahn-Hilliard equation for the sole $\phi$ with a dynamic boundary condition. The upper semicontinuity of the global attractor is also analyzed. The paper extends and revisits some results previously obtained by A. Miranville et al.

Mathematics Subject Classification (2000). Primary 35B41, 35K55, 37L30; Secondary 80A22.

Keywords. Phase-field equations, non-isothermal Allen-Cahn systems, dynamic boundary conditions, global attractors, exponential attractors.
\end{abstract}

\section{Introduction}

A well-known mathematical model which describes phase transitions in presence of temperature variations, but in absence of mechanical stresses, is the phase-field system (see [7], cf. also [5,25])

$$
\begin{aligned}
\delta \partial_{t} \phi-\Delta \phi+f_{1}(\phi)-\lambda u & =0, \\
\varepsilon \partial_{t} u+\lambda \partial_{t} \phi-\Delta u & =0,
\end{aligned}
$$

in $\Omega \times(0,+\infty), \Omega$ being a bounded domain in $\mathbb{R}^{3}$ with smooth boundary $\Gamma$. The variable $\phi(x, t)$ represents the order parameter (or phase-field), while $u(x, t)$ stands for the (relative) temperature. The given parameters $\delta$ and $\varepsilon$ are positive, while the constant $\lambda$ represents the latent heat. Moreover, the function $f_{1}$ is the derivative of a double-well like potential which accounts for the presence of different phases. Of course, $\Delta$ is the spatial Laplace operator. 
There is a consistent literature on the mathematical analysis of system (1.1)(1.2) when $\phi$ is subject to homogeneous Neumann (or Dirichlet) boundary conditions. In particular, the associated dissipative dynamical system has been analyzed in details as well as the convergence to steady states (see, e.g., $[1-4,10,12,17,20$ $23,34,35,39])$. It is also worth observing that system (1.1)-(1.2) can be viewed as a singular perturbation of the celebrated Cahn-Hilliard equation that accounts for phase separation dynamics (see, e.g., [28,29] and references therein). In fact, if we formally set $\varepsilon=0$ in equation (1.2), then we can easily deduce the (viscous) Cahn-Hilliard equation

$$
\lambda^{2} \partial_{t} \phi-\Delta\left(\delta \partial_{t} \phi-\Delta \phi+f_{1}(\phi)\right)=0,
$$

in $\Omega \times(0,+\infty)$, which reduces to the classical Cahn-Hilliard equation when $\delta=0$ (see [6], cf. also [33,36]). More recently, system (1.1)-(1.2) has been endowed with a dynamic boundary condition for $\phi$ which accounts for possible interactions of the material with the walls (see [19], cf. also $[8,9,11,18,26,30,32,38]$ and references therein). This condition reads

$$
\partial_{t} \phi=\alpha \Delta_{\Gamma} \phi-\partial_{\mathbf{n}} \phi-\beta \phi-f_{2}(\phi),
$$

on $\Gamma \times(0,+\infty)$. Here $\alpha$ and $\beta$ are given positive constants, $\Delta_{\Gamma}$ denotes the LaplaceBeltrami operator on the surface $\Gamma, \partial_{\mathbf{n}}$ stands for the outward normal derivative, and $f_{2}$ is a given function satisfying suitable assumptions. The corresponding problem, with $u$ subject to homogeneous Neumann conditions, has been interpreted and studied in [19] as a dissipative dynamical system, proving the existence of families of exponential attractors $\left\{\mathcal{M}_{\varepsilon}\right\}$ and global attractors $\left\{\mathcal{A}_{\varepsilon}\right\}$. The authors have also shown the uniformity of the former with respect to $\varepsilon$ as well as the upper semicontinuity of the latter as $\varepsilon$ goes to 0 . Here we want to complete their analysis by proving the robustness of $\left\{\mathcal{M}_{\varepsilon}\right\}$, i.e., we obtain the explicit control of the Hausdorff distance between $\mathcal{M}_{\varepsilon}$ and $\mathcal{M}_{0}$ by a constant times some power of $\varepsilon$. This result says that the nontransient dynamics of the phase-field system (1.1)-(1.2) with the dynamic boundary condition (1.4) is close to the one of equation (1.3) subject to (1.4) (for similar results see, e.g., [26,27] and references therein). In addition, we will also give a slight generalization of the upper semicontinuity result. We recall that the upper (and lower) semicontinuity of the global attractor for a phase-field system like (1.1)-(1.2) endowed with standard boundary conditions has been already analyzed in [13-16]. However, only in [27] the existence of a robust family of exponential attractors is established, provided that $u$ and $\phi$ satisfy homogeneous Dirichlet (or Neumann) boundary conditions. We will follow a similar strategy.

Summing up, we are concerned with the study of the following boundary value problem:

$$
\begin{aligned}
& \left\{\begin{array}{l}
\delta \partial_{t} \phi=\Delta \phi-f_{1}(\phi)+\lambda u+g_{1}, \text { in } \Omega \times(0,+\infty), \\
\partial_{t} \phi=\alpha \Delta_{\Gamma} \phi-\partial_{\mathbf{n}} \phi-\beta \phi-f_{2}(\phi)+g_{2}, \text { on } \Gamma \times(0,+\infty),
\end{array}\right. \\
& \left\{\begin{array}{l}
\varepsilon \partial_{t} u-\Delta u=-\lambda \partial_{t} \phi, \text { in } \Omega \times(0,+\infty), \\
\partial_{\mathbf{n}} u=0, \text { on } \Gamma \times(0,+\infty),
\end{array}\right.
\end{aligned}
$$


endowed with the initial conditions

$$
\phi_{\mid t=0}=\phi_{0}, \quad u_{\mid t=0}=u_{0} .
$$

Here $g_{1}$ and $g_{2}$ are given external forces. The limiting equations of the above problem are formally obtained by taking $\varepsilon=0$. This yields

$$
\begin{aligned}
& \left\{\begin{array}{l}
\delta \partial_{t} \bar{\phi}_{0}=\Delta \bar{\phi}_{0}-f_{1}\left(\bar{\phi}_{0}\right)+\lambda \bar{u}_{0}+g_{1}, \text { in } \Omega \times(0,+\infty), \\
\partial_{t} \bar{\phi}_{0}=\alpha \bar{\nu}_{\Gamma} \bar{\phi}_{0}-\partial_{\mathbf{n}} \bar{\phi}_{0}-\beta \bar{\phi}_{0}-f_{2}\left(\bar{\phi}_{0}\right)+g_{2}, \text { on } \Gamma \times(0,+\infty),
\end{array}\right. \\
& \left\{\begin{array}{l}
\lambda \partial_{t} \bar{\phi}_{0}=\Delta \bar{u}_{0}, \quad \text { in } \Omega \times(0,+\infty), \\
\partial_{\mathbf{n}} \bar{u}_{0}=0, \text { in } \Gamma \times(0,+\infty),
\end{array}\right.
\end{aligned}
$$

with initial condition

$$
\bar{\phi}_{0 \mid t=0}=\phi_{0} .
$$

Note that (1.3) can replace the first of (1.8) and (1.9). Moreover, $\bar{\phi}_{0}$ also fulfills the boundary condition

$$
\partial_{\mathbf{n}}\left(\delta \partial_{t} \bar{\phi}_{0}-\Delta \bar{\phi}_{0}+f_{1}\left(\bar{\phi}_{0}\right)-g_{1}\right)=0,
$$

on $\Gamma \times(0,+\infty)$. Therefore, the spatial average of $\bar{\phi}_{0}$ is conserved.

The paper is organized as follows. In Section 2 we recall some existence results and several useful estimates proved in [19] (see also [18]) and state the main result, i.e., the existence of a robust family of exponential attractors. Section 3 is devoted to the most crucial step, namely, estimates on the difference between the solutions to problem (1.5)-(1.7) and problem (1.8)-(1.10). This result allows us to prove in Section 4 the existence of a family of exponential attractors that is robust with respect to $\varepsilon$. Finally, in Section 5, we slightly extend the result of [19] on the upper semicontinuity of the global attractors $\mathcal{A}_{\varepsilon}$ at $\varepsilon=0$, taking a larger phase-space.

\section{Preliminaries and main results}

Following $[19,26]$, it is convenient to introduce an additional variable $\psi:=\phi_{\mid \Gamma}$ and to interpret the dynamic boundary condition (1.5) as an evolution equation on the boundary $\Gamma$. Hence, for any $\varepsilon \in(0,1]$, problem (1.5)-(1.7) becomes

Problem $\mathcal{P}_{\varepsilon}$. Find $(\phi, \psi, u)$ such that

$$
\begin{aligned}
& \left\{\begin{array}{l}
\delta \partial_{t} \phi=\Delta \phi-f_{1}(\phi)+\lambda u+g_{1}, \quad \text { in } \Omega \times(0,+\infty), \\
\partial_{t} \psi=\alpha \Delta_{\Gamma} \psi-\partial_{\mathbf{n}} \phi-\beta \psi-f_{2}(\psi)+g_{2}, \quad \text { on } \Gamma \times(0,+\infty), \\
\psi=\phi_{\mid \Gamma},
\end{array}\right. \\
& \left\{\begin{array}{l}
\varepsilon \partial_{t} u-\Delta u=-\lambda \partial_{t} \phi, \text { in } \Omega \times(0,+\infty), \\
\partial_{\mathbf{n}} u=0, \text { on } \Gamma \times(0,+\infty),
\end{array}\right.
\end{aligned}
$$

with the initial conditions

$$
\phi_{\mid t=0}=\phi_{0}, \quad \psi_{\mid t=0}=\psi_{0}, \quad u_{\mid t=0}=u_{0} .
$$


Observe that, due to the boundary conditions (2.2), the enthalpy is conserved, namely,

$$
I_{\varepsilon}:=\varepsilon\langle u(t)\rangle+\lambda\langle\phi(t)\rangle=\varepsilon\left\langle u_{0}\right\rangle+\lambda\left\langle\phi_{0}\right\rangle,
$$

for any $t \geq 0$, where $\langle v\rangle$ denotes the spatial average of a function $v$ on $\Omega$.

Let us introduce the Hilbert spaces

$$
\mathbb{V}_{s}:=H^{s}(\Omega) \times H^{s}(\Gamma) \times H^{s}(\Omega),
$$

for any $s \in \mathbb{N}$. The spaces $H^{s}(\Omega)$ and $H^{s}(\Gamma)$ are endowed with the norms induced by their standard inner products and are denoted by $\|\cdot\|_{H^{s}(\Omega)}$ and $\|\cdot\|_{H^{s}(\Gamma)}$, if $s>0$, while, if $s=0$, by $\|\cdot\|_{2}$ and $\|\cdot\|_{2, \Gamma}$, respectively. In particular, $\langle\cdot, \cdot\rangle_{2}$ denotes the standard scalar product in $L^{2}(\Omega)$. Then (see $[19,26]$ ) we introduce the function space

$$
\mathbb{D}_{\varepsilon}^{M}:=\left\{(\phi, \psi, u) \in \mathbb{V}_{2}: \psi=\phi_{\mid \Gamma}, \quad\left(\partial_{\mathbf{n}} u\right)_{\mid \Gamma}=0, \quad\left|I_{\varepsilon}\right| \leq M\right\},
$$

for any given $\varepsilon \in(0,1]$ and any fixed $M \geq 0$. This space is a complete metric space endowed with the metric induced by the $\mathbb{V}_{2}$-norm.

We assume that

$$
g_{1} \in L^{2}(\Omega), \quad g_{2} \in L^{2}(\Gamma),
$$

while, concerning the nonlinear functions $f_{i}: \mathbb{R} \rightarrow \mathbb{R}$, we assume that they belong to $C^{\prime}(\mathbb{R})$ and satisfy the conditions

$$
\begin{aligned}
\lim _{|y| \rightarrow+\infty} \inf f_{i}^{\prime}(y) & >0, \\
f_{i}(y) y & \geq \nu_{i} y^{2}-\nu_{i}^{\prime},
\end{aligned}
$$

for some positive $\nu_{1}$ and some nonnegative $\nu_{2}, \nu_{1}^{\prime}, \nu_{2}^{\prime}$. In addition, we assume the local Lipschitz continuity of $f_{i}^{\prime}, i=1,2$.

On account of [19, Lemma 2.1] (see also [27, Lemma 1.3]), the following a priori estimate can be proven.

Theorem 1. Let assumptions (2.6)-(2.8) be satisfied. Then, every sufficiently smooth solution $(\phi(t), \psi(t), u(t))$ to $\mathcal{P}_{\varepsilon}$ satisfies the following estimate:

$$
\begin{gathered}
\|(\phi(t), \psi(t), u(t))\|_{\mathbb{V}_{2}}^{2}+\varepsilon^{2}\left\|\partial_{t} u(t)\right\|_{2}^{2}+\left\|\partial_{t} \phi(t)\right\|_{2}^{2}+\left\|\partial_{t} \psi(t)\right\|_{2, \Gamma}^{2} \\
\quad+\int_{t}^{t+1}\left(\left\|\partial_{t} \phi(s)\right\|_{H^{1}(\Omega)}^{2}+\left\|\partial_{t} \psi(s)\right\|_{H^{1}(\Gamma)}^{2}\right) d s \\
\leq Q_{1}\left(\left\|\left(\phi_{0}, \psi_{0}, u_{0}\right)\right\|_{\mathbb{V}_{2}}^{2}\right) e^{-\rho t}+Q_{1}\left(\left\|g_{1}\right\|_{2}^{2}+\left\|g_{1}\right\|_{2, \Gamma}^{2}\right),
\end{gathered}
$$

where $\rho>0$ and the positive and monotone increasing function $Q_{1}$ are independent of $\varepsilon$.

Existence and uniqueness for $\mathcal{P}_{\varepsilon}$ have also been proved in [19] (see also [18] for a slightly more general result). Clearly, Theorem 1 entails that the corresponding semiflow has a bounded absorbing set in $\mathbb{V}_{2}$. 
Theorem 2. Let assumptions (2.6)-(2.8) be satisfied. Then, for every $\left(\phi_{0}, \psi_{0}, u_{0}\right) \in$ $\mathbb{D}_{\varepsilon}^{M}$, problem $\mathcal{P}_{\varepsilon}$ has a unique solution $(\phi(t), \psi(t), u(t)) \in C\left([0,+\infty), \mathbb{D}_{\varepsilon}^{M}\right)$ which satisfies estimate (2.9). Consequently, $\mathcal{P}_{\varepsilon}$ defines a semiflow $S_{t}^{\varepsilon}: \mathbb{D}_{\varepsilon}^{M} \rightarrow \mathbb{D}_{\varepsilon}^{M}$ defined by

$$
S_{t}^{\varepsilon}\left(\phi_{0}, \psi_{0}, u_{0}\right):=(\phi(t), \psi(t), u(t)), \quad \forall t \geq 0 .
$$

Let us now consider the limiting problem $\mathcal{P}_{0}$ which can be formulated as

Problem $\mathcal{P}_{0}$. Find $\left(\bar{\phi}_{0}, \bar{\psi}_{0}, \bar{u}_{0}\right)$ such that

$$
\begin{aligned}
& \left\{\begin{array}{l}
\delta \partial_{t} \bar{\phi}_{0}=\Delta \bar{\phi}_{0}-f_{1}\left(\bar{\phi}_{0}\right)+\lambda \bar{u}_{0}+g_{1}, \text { in } \Omega \times(0,+\infty), \\
\partial_{t} \bar{\psi}_{0}=\alpha \Delta_{\Gamma} \bar{\psi}_{0}-\partial_{\mathbf{n}} \bar{\phi}_{0}-\beta \bar{\psi}_{0}-f_{2}\left(\bar{\psi}_{0}\right)+g_{2}, \text { on } \Gamma \times(0,+\infty), \\
\bar{\psi}_{0}=\bar{\phi}_{0 \mid \Gamma},
\end{array}\right. \\
& \left\{\begin{array}{l}
\lambda \partial_{t} \bar{\phi}_{0}=\Delta \bar{u}_{0}, \quad \text { in } \Omega \times(0,+\infty), \\
\partial_{\mathbf{n}} \bar{u}_{0}=0, \text { on } \Gamma \times(0,+\infty),
\end{array}\right.
\end{aligned}
$$

with the initial conditions

$$
\bar{\phi}_{0 \mid t=0}=\phi_{0}, \quad \bar{\psi}_{0 \mid t=0}=\psi_{0}
$$

Observe that, from the first equations of (2.11) and (2.12), it follows that

$$
\left\{\begin{array}{l}
-\delta \Delta \bar{u}_{0}+\lambda^{2} \bar{u}_{0}=-\lambda\left(\Delta \bar{\phi}_{0}-f_{1}\left(\bar{\phi}_{0}\right)+g_{1}\right), \\
\partial_{\mathbf{n}} \bar{u}_{0}=0 .
\end{array}\right.
$$

Therefore $\bar{u}_{0}(t)$ is uniquely defined by $(2.14)$, provided that $\bar{\phi}_{0}(t)$ is known. Then, by standard elliptic estimates, it follows that there exists a nonlinear operator (see [27])

$$
\mathcal{L} \in C^{1}\left(H^{2}(\Omega),\left\{v \in H^{2}(\Omega): \partial_{\mathbf{n}} v=0\right\}\right),
$$

such that, for any $t \geq 0$ and any $\bar{\phi}_{0}(t) \in H^{2}(\Omega)$,

$$
\bar{u}_{0}(t)=\mathcal{L}\left(\bar{\phi}_{0}(t)\right) .
$$

Consequently, the solution to $\mathcal{P}_{0}$ exists only for initial data $\left(\phi_{0}, \psi_{0}, u_{0}\right)$ belonging to the infinite dimensional submanifold $\mathbb{L}^{M}$ of the phase space $\mathbb{V}_{2}$ defined by

$$
\begin{aligned}
\mathbb{L}^{M}:=\left\{\left(\phi_{0}, \psi_{0}, u_{0}\right) \in \mathbb{V}_{2}: \psi_{0}=\phi_{0 \mid \Gamma}, u_{0}=\mathcal{L}\left(\phi_{0}\right),\right. & \\
& \left.\partial_{\mathbf{n}} u_{0}=0,\left|\lambda\left\langle\phi_{0}\right\rangle\right| \leq M\right\} .
\end{aligned}
$$

The following theorem is a direct consequence of the results in $[19,27]$.

Theorem 3. Let assumptions (2.6) -(2.8) be satisfied. Then, for every $\left(\phi_{0}, \psi_{0}, u_{0}\right) \in$ $\mathbb{L}^{M}$, problem $\mathcal{P}_{0}$ has a unique solution $\left(\bar{\phi}_{0}(t), \bar{\psi}_{0}(t), \bar{u}_{0}(t)\right) \in C\left([0,+\infty) ; \mathbb{L}^{M}\right)$ which 
satisfies the following estimate:

$$
\begin{gathered}
\left\|\left(\bar{\phi}_{0}(t), \bar{\psi}_{0}(t), \bar{u}_{0}(t)\right)\right\|_{\mathbb{V}_{2}}^{2}+\left\|\partial_{t} \bar{u}_{0}(t)\right\|_{2}^{2}+\left\|\partial_{t} \bar{\phi}_{0}(t)\right\|_{2}^{2}+\left\|\partial_{t} \bar{\psi}_{0}(t)\right\|_{2, \Gamma}^{2} \\
\quad+\int_{t}^{t+1}\left(\left\|\left(\partial_{t} \bar{\phi}_{0}(s), \partial_{t} \bar{\psi}_{0}(s), \partial_{t} \bar{u}_{0}(s)\right)\right\|_{\mathbb{V}_{1}}^{2}+\left\|\partial_{t}^{2} \bar{u}_{0}(s)\right\|_{\left(H^{1}(\Omega)\right)^{*}}^{2}\right) d s \\
\leq Q_{2}\left(\left\|\left(\phi_{0}, \psi_{0}, u_{0}\right)\right\|_{\mathbb{V}_{2}}^{2}\right) e^{-\rho t}+Q_{2}\left(\left\|g_{1}\right\|_{2}^{2}+\left\|g_{1}\right\|_{2, \Gamma}^{2}\right)
\end{gathered}
$$

for some $\rho>0$ and some positive and monotone increasing function $Q_{2}$. Consequently, $\mathcal{P}_{0}$ defines a semiflow $S_{t}^{0}$ on the manifold $\mathbb{L}^{M}$ by setting

$$
S_{t}^{0}: \mathbb{L}^{M} \rightarrow \mathbb{L}^{M}, \quad S_{t}^{0}\left(\phi_{0}, \psi_{0}, u_{0}\right):=\left(\bar{\phi}_{0}(t), \bar{\psi}_{0}(t), \bar{u}_{0}(t)\right) .
$$

Let us now introduce the projection $\mathbb{P}: \mathbb{L}^{M} \rightarrow \widehat{\mathbb{L}}^{M}$, by setting $\mathbb{P}\left(\phi_{0}, \psi_{0}, u_{0}\right)=$ $\left(\phi_{0}, \psi_{0}\right)$, where

$$
\widehat{\mathbb{L}}^{M}:=\left\{\left(\phi_{0}, \psi_{0}\right) \in H^{2}(\Omega) \times H^{2}(\Gamma): \psi_{0}=\phi_{0 \mid \Gamma},\left|\lambda\left\langle\phi_{0}\right\rangle\right| \leq M\right\} .
$$

Then, we define a semiflow $\widehat{S}_{t}^{0}$ on the complete metric space $\widehat{\mathbb{L}}^{M}$ by setting

$$
\widehat{S}_{t}^{0}: \widehat{\mathbb{L}}^{M} \rightarrow \widehat{\mathbb{L}}^{M}, \quad \widehat{S}_{t}^{0}\left(\phi_{0}, \psi_{0}\right):=\left(\bar{\phi}_{0}(t), \bar{\psi}_{0}(t)\right),
$$

where $\left(\bar{\phi}_{0}(t), \bar{\psi}_{0}(t)\right)$ is the unique solution to (1.3)-(1.4) and (1.11) with $\bar{\psi}_{0}=$ $\bar{\phi}_{0 \mid \Gamma}$. This is nothing but the dynamical system associated with the viscous CahnHilliard equation subject to no-flux and dynamic boundary conditions. It is clear that $\mathbb{P} S_{t}^{0}=\widehat{S}_{t}^{0}$. On the other hand, $S_{t}^{0}$ can be obtained from $\widehat{S}_{t}^{0}$ by a lifting of $\widehat{\mathbb{L}}^{M}$ to $\mathbb{L}^{M}$ defined through (2.14). It is known that $\left(\widehat{S}_{t}^{0}, \widehat{\mathbb{L}}^{M}\right)$ is a dissipative dynamical system and possesses an exponential attractor $\widehat{\mathcal{M}}_{0}^{M}$ which is contained in a bounded subset of $H^{3}(\Omega) \times H^{3}(\Gamma)$ (see [27]). Correspondingly, we set

$$
\mathcal{M}_{0}^{M}:=\left\{(\phi, \psi, u) \in \mathbb{L}^{M}:(\phi, \psi) \in \widehat{\mathcal{M}}_{0}^{M}, u=\mathcal{L}(\phi)\right\} .
$$

We are now ready to state the main result of this paper.

Theorem 4. Let assumptions (2.6)-(2.8) be satisfied. Then, for every fixed $M \geq 0$, there exists a family of compact sets $\mathcal{M}_{\varepsilon}^{M} \subset \mathbb{D}_{\varepsilon}^{M}$, where $\varepsilon \in[0,1]$ and $\mathbb{D}_{0}^{M}:=\mathbb{L}^{M}$, with the following properties:

(i) The sets $\mathcal{M}_{\varepsilon}^{M}$ are semi-invariant with respect to the semiflows $S_{t}^{\varepsilon}$ associated with problem $\mathcal{P}_{\varepsilon}$, that is,

$$
S_{t}^{\varepsilon}\left(\mathcal{M}_{\varepsilon}^{M}\right) \subseteq \mathcal{M}_{\varepsilon}^{M}, \quad \forall t \geq 0 .
$$

(ii) The fractal dimension of the sets $\mathcal{M}_{\varepsilon}^{M}$ is finite and uniformly bounded with respect to $\varepsilon$, i.e.,

$$
\operatorname{dim}_{F}\left(\mathcal{M}_{\varepsilon}^{M}, \mathbb{D}_{\varepsilon}^{M}\right) \leq C_{M}<+\infty,
$$

where $C_{M}$ is independent of $\varepsilon$. 
(iii) Each $\mathcal{M}_{\varepsilon}^{M}$ attracts exponentially any bounded subset of $\mathbb{D}_{\varepsilon}^{M}$, that is, there exist a positive constant $\rho$ and a monotonic nonnegative function $Q$, depending on $M$ but independent of $\varepsilon$, such that, for every bounded subset $B$ of $\mathbb{D}_{\varepsilon}^{M}$, we have

$$
\operatorname{dist}_{\mathbb{D}_{\varepsilon}^{M}}\left(S_{t}^{\varepsilon} B, \mathcal{M}_{\varepsilon}^{M}\right) \leq Q\left(\|B\|_{\mathbb{D}_{\varepsilon}^{M}}\right) e^{-\rho t},
$$

where $\operatorname{dist}_{\mathbb{D}_{\varepsilon}^{M}}(X, Y):=\sup _{x \in X} \inf _{y \in Y}\|x-y\|_{\mathbb{V}_{2}}$ is the Hausdorff semidistance.

(iv) There exist positive constants $K$ and $\kappa \in(0,1)$, depending on $M$ but independent of $\varepsilon$, such that

$$
\operatorname{dist}_{\mathbb{D}_{\varepsilon}^{M}}^{\text {symm }}\left(\mathcal{M}_{0}^{M}, \mathcal{M}_{\varepsilon}^{M}\right) \leq K \varepsilon^{\kappa},
$$

where $\operatorname{dist}_{\mathbb{D}_{\varepsilon}^{M}}^{\text {symm }}(X, Y):=\max \left\{\operatorname{dist}_{\mathbb{D}_{\varepsilon}^{M}}(X, Y)\right.$, dist $\left.t_{\mathbb{D}_{\varepsilon}^{M}}(Y, X)\right\}$ is the Hausdorff distance.

Remark 1. Let us recall that the only novelty here is property $(i v)$ and its proof since the rest was already proven in [18, Thm. 4.2].

\section{Estimates on the difference of solutions}

The main goal of this section is to estimate the difference of the solutions to problems $\mathcal{P}_{\varepsilon}$ and $\mathcal{P}_{0}$. This is a very crucial step in order to prove property $(i v)$ of Theorem 4 (cf. next section) and it has an interest on its own.

We argue as in [27] using the boundary layer technique devised in [37]. Thus we start by computing the first terms of the asymptotic expansions of the solution $(\phi(t), \psi(t), u(t))$ of problem $\mathcal{P}_{\varepsilon}$ as $\varepsilon \rightarrow 0$. This is done by introducing the fast variable $\tau:=t / \varepsilon$ and expanding this solution as follows:

$$
\left\{\begin{array}{l}
\phi(t)=\phi_{0}(t, \tau)+\varepsilon \phi_{1}(t, \tau)+\cdots \\
\psi(t)=\psi_{0}(t, \tau)+\varepsilon \psi_{1}(t, \tau)+\cdots \\
u(t)=u_{0}(t, \tau)+\varepsilon u_{1}(t, \tau)+\cdots
\end{array}\right.
$$

where $\phi_{i}(t, \tau), \psi_{i}(t, \tau)$ and $u_{i}(t, \tau)$ are functions (independent of $\varepsilon$ ) of the form

$$
\phi_{i}(t, \tau)=\bar{\phi}_{i}(t)+\widetilde{\phi}_{i}(\tau), \quad \psi_{i}(t, \tau)=\bar{\psi}_{i}(t)+\widetilde{\psi}_{i}(\tau), \quad u_{i}(t, \tau)=\bar{u}_{i}(t)+\widetilde{u}_{i}(\tau),
$$

with

$$
\lim _{\tau \rightarrow+\infty} \widetilde{\phi}_{i}(\tau)=\lim _{\tau \rightarrow+\infty} \widetilde{\psi}_{i}(\tau)=\lim _{\tau \rightarrow+\infty} \widetilde{u}_{i}(\tau)=0
$$

Inserting expansions (3.1) into equations (2.1)-(2.2), we obtain some equations for the terms $\phi_{i}(t, \tau), \psi_{i}(t, \tau)$ and $u_{i}(t, \tau)$. Indeed, at order $\varepsilon^{-1}$, it follows from equation (2.1) that

$$
\delta \partial_{\tau} \widetilde{\phi}_{0}(\tau)=0, \quad \partial_{\tau} \widetilde{\psi}_{0}(\tau)=0 .
$$

Consequently, by $(3.3)$, we have $\widetilde{\phi}_{0}(\tau)=0, \widetilde{\psi}_{0}(\tau)=0$. At order $\varepsilon$, equations $(2.1)$ yield

$$
\left\{\begin{array}{l}
\delta \partial_{t} \bar{\phi}_{0}(t)=\Delta \bar{\phi}_{0}(t)-f_{1}\left(\bar{\phi}_{0}(t)\right)+\lambda \bar{u}_{0}(t)+g_{1} \\
\partial_{t} \bar{\psi}_{0}(t)=\alpha \Delta_{\Gamma} \bar{\psi}_{0}(t)-\partial_{\mathbf{n}} \bar{\phi}_{0}(t)-\beta \bar{\psi}_{0}(t)-f_{2}\left(\bar{\psi}_{0}(t)\right)+g_{2} .
\end{array}\right.
$$


On the other hand, we deduce from equation (2.2) that

$$
\left\{\begin{array}{l}
\lambda \partial_{t} \bar{\phi}_{0}(t)=\Delta \bar{u}_{0}(t) \\
\partial_{\mathbf{n}} \bar{u}_{0}(t)=0
\end{array}\right.
$$

and

$$
\left\{\begin{array}{l}
\partial_{\tau} \widetilde{u}_{0}(\tau)-\Delta \widetilde{u}_{0}(\tau)=-\lambda \partial_{\tau} \widetilde{\phi}_{1}(\tau) \\
\partial_{\mathbf{n}} \widetilde{u}_{0}(\tau)=0
\end{array}\right.
$$

with

$$
\delta \partial_{\tau} \widetilde{\phi}_{1}(\tau)=\lambda \widetilde{u}_{0}(\tau), \quad \partial_{\tau} \widetilde{\psi}_{1}(\tau)=0
$$

Consequently, on account of (3.3), the remaining boundary layer terms are found from

$$
\widetilde{\phi}_{1}(\tau)=\frac{\lambda}{\delta} \int_{\tau}^{\infty} \widetilde{u}_{0}(s) d s, \quad \widetilde{\psi}_{1}(\tau)=0 .
$$

Expanding now the initial data, we have

$$
\left\{\begin{array}{l}
\bar{\phi}_{1}(0)+\widetilde{\phi}_{1}(0)=0, \widetilde{\phi}_{0}(0)=0, \bar{\phi}_{0}(0)=\phi(0), \\
\widetilde{\psi}_{0}(0)=\bar{\psi}_{1}(0)=\widetilde{\psi}_{1}(0)=0, \bar{\psi}_{0}(0)=\psi(0),
\end{array}\right.
$$

and

$$
\bar{u}_{1}(0)+\widetilde{u}_{1}(0)=0, \quad \widetilde{u}_{0}(0)=u(0)-\bar{u}_{0}(0) .
$$

Hence, the function $\left(\bar{\phi}_{0}(t), \bar{\psi}_{0}(t), \bar{u}_{0}(t)\right)$ solves $(3.4)-(3.5)$ with initial data $\bar{\phi}_{0}(0)=$ $\phi(0), \bar{\psi}_{0}(0)=\psi(0)$, that is,

$$
\left(\bar{\phi}_{0}(t), \bar{\psi}_{0}(t), \bar{u}_{0}(t)\right)=S_{t}^{0}(\phi(0), \psi(0), \mathcal{L}(\phi(0))) .
$$

Recalling [27], we observe that it suffices to seek for a solution of problem $\mathcal{P}_{\varepsilon}$ of the form

$$
\left\{\begin{array}{l}
\phi(t)=\bar{\phi}_{0}(t)+\varepsilon \widetilde{\phi}(\tau)+\varepsilon \widehat{\phi}(t) \\
\psi(t)=\bar{\psi}_{0}(t)+\varepsilon \widehat{\psi}(t) \\
u(t)=\bar{u}_{0}(t)+\widetilde{u}(\tau)+\varepsilon \widehat{u}(t)
\end{array}\right.
$$

where $\left(\bar{\phi}_{0}(t), \bar{\psi}_{0}(t), \bar{u}_{0}(t)\right)$ is given by $(3.9)$, the boundary layer term $\widetilde{u}(\tau)$ solves

$$
\left\{\begin{array}{l}
\partial_{\tau} \widetilde{u}(\tau)-\Delta \widetilde{u}(\tau)+\frac{\lambda^{2}}{\delta} \widetilde{u}(\tau)=0 \\
\partial_{\mathbf{n}} \widetilde{u}(\tau)=0 \\
\widetilde{u}(0)=u(0)-\mathcal{L}(\phi(0))
\end{array}\right.
$$

and the boundary layer terms $\widetilde{\phi}(\tau)$ and $\widetilde{\psi}(\tau)$ are defined by $(3.7)$ (where $\widetilde{u}_{0}(\tau)$, $\widetilde{\phi}_{1}(\tau), \widetilde{\psi}_{1}(\tau)$ are replaced by $\widetilde{u}(\tau), \widetilde{\phi}(\tau)$ and $\widetilde{\psi}(\tau)$, respectively). Moreover, the 
boundary layer terms $\widehat{\phi}(t), \widehat{\psi}(t)$ and $\widehat{u}(t)$ satisfy the following problems:

$$
\left\{\begin{aligned}
\delta \partial_{t} \widehat{\phi}(t)= & \Delta \widehat{\phi}(t)-\frac{1}{\varepsilon}\left[f_{1}\left(\bar{\phi}_{0}(t)+\varepsilon \widetilde{\phi}(\tau)+\varepsilon \widehat{\phi}(t)\right)-f_{1}\left(\bar{\phi}_{0}(t)\right)\right] \\
& +\lambda \widehat{u}(t)+\Delta \widetilde{\phi}(\tau), \\
& \partial_{t} \widehat{\psi}(t)=\alpha \Delta_{\Gamma} \widehat{\psi}(t)-\partial_{\mathbf{n}} \widehat{\phi}(t)-\beta \widehat{\psi}(t) \\
& -\frac{1}{\varepsilon}\left[f_{2}\left(\bar{\psi}_{0}(t)+\varepsilon \widehat{\psi}(t)\right)-f_{2}\left(\bar{\psi}_{0}\right)\right]-\partial_{\mathbf{n}} \widetilde{\phi}(\tau), \\
& \widehat{\phi}(0)=-\widetilde{\phi}(0), \quad \widehat{\psi}(0)=0,
\end{aligned}\right.
$$

and

$$
\left\{\begin{array}{l}
\varepsilon \partial_{t} \widehat{u}(t)=\Delta \widehat{u}(t)+\partial_{t} \widehat{\phi}(t)-\partial_{t} \bar{u}_{0}(t), \\
\partial_{\mathbf{n}} \widehat{u}(t)=0 \\
\widehat{u}(0)=0
\end{array}\right.
$$

The next lemma provides some estimates on the boundary layer terms $\widetilde{u}(\tau)$ and $\widetilde{\phi}(\tau)$, given by (3.11) and (3.7), respectively. The result is a straightforward consequence of standard energy estimates.

Lemma 5. The following estimates hold:

$$
\begin{aligned}
\|\widetilde{u}(\tau)\|_{H^{2}(\Omega)}+\left\|\partial_{\tau} \widetilde{u}(\tau)\right\|_{2} & \leq C\|\widetilde{u}(0)\|_{H^{2}(\Omega)} e^{-\rho \tau}, \\
\|\widetilde{\phi}(\tau)\|_{H^{2}(\Omega)}+\left\|\partial_{\tau} \widetilde{\phi}(\tau)\right\|_{H^{2}(\Omega)} & \leq C\|\widetilde{u}(0)\|_{H^{2}(\Omega)} e^{-\rho \tau},
\end{aligned}
$$

where $\rho, C>0$ are both independent of $\varepsilon$.

We can now estimate the remainder terms $\widehat{\phi}(t), \widehat{\psi}(t)$ and $\widehat{u}(t)$ in expansion (3.10).

Lemma 6. The following estimate holds:

$$
\|(\widehat{\phi}(t), \widehat{\psi}(t), \widehat{u}(t))\|_{\mathbb{V}_{2}}^{2}+\left\|\partial_{t} \widehat{\phi}(t)\right\|_{2}^{2}+\left\|\partial_{t} \widehat{\psi}(t)\right\|_{2, \Gamma}^{2}+\varepsilon\left\|\partial_{t} \widehat{u}(t)\right\|_{2}^{2} \leq C e^{L t},
$$

where the positive constants $C$ and $L$ depend on $\|(\phi(0), \psi(0), u(0))\|_{\mathbb{V}_{2}}$, but are independent of $\varepsilon$.

Proof. We first note that the functions $\widetilde{\phi}(\tau), \varepsilon \widehat{\phi}(t)$ and $\varepsilon \widehat{\psi}(t)$ are uniformly bounded with respect to $\varepsilon$ in $H^{2}(\Omega), H^{2}(\Omega)$ and $H^{2}(\Gamma)$, respectively. This easily follows from estimates (3.14)-(3.15), (2.9), (2.18). In particular, the initial datum $\widetilde{\phi}(0)$ is uniformly bounded in $H^{2}(\Omega)$ as $\varepsilon \rightarrow 0$. Observe preliminarily that all the constants $C_{i}$ are independent of $\varepsilon$. Moreover, note that (2.7) entails

$$
f_{i}^{\prime}(y) \geq-K_{i}, \quad \forall y \in \mathbb{R},
$$

for some $K_{i}>0, i=1,2$.

Multiplying the first equation of (3.12) by $\partial_{t} \widehat{\phi}(t)$, integrating over $\Omega$, and using the second equation of (3.12), we have

$$
\begin{gathered}
\partial_{t}\left(\|\nabla \widehat{\phi}(t)\|_{2}^{2}+\alpha\left\|\nabla_{\Gamma} \widehat{\psi}(t)\right\|_{2, \Gamma}^{2}+\beta\|\widehat{\psi}(t)\|_{2, \Gamma}^{2}\right)+2 \delta\left\|\partial_{t} \widehat{\phi}(t)\right\|_{2}^{2}+2\left\|\partial_{t} \widehat{\psi}(t)\right\|_{2, \Gamma}^{2} \\
\leq C_{1}\left(\|\widehat{\phi}(t)\|_{H^{1}(\Omega)}^{2}+\|\widehat{\psi}(t)\|_{2, \Gamma}^{2}\right)+C_{2}\left(\|\widetilde{\phi}(\tau)\|_{H^{2}(\Omega)}^{2}+\|\widehat{u}(t)\|_{2}^{2}\right),
\end{gathered}
$$


where $C_{1}>0$ only depends on $K_{i}$ and $C_{2}>0$ only depends on $\|\widetilde{\phi}(0)\|_{H^{2}(\Omega)}$. We now differentiate both equations of (3.12) with respect to $t$, multiply the first equation by $\partial_{t} \widehat{\phi}(t)$ and integrate over $\Omega$ to obtain

$$
\begin{aligned}
\partial_{t}\left(\delta\left\|\partial_{t} \widehat{\phi}(t)\right\|_{2}^{2}+\left\|\partial_{t} \widehat{\psi}(t)\right\|_{2, \Gamma}^{2}\right)+2\left\|\partial_{t} \widehat{\phi}(t)\right\|_{H^{1}(\Omega)}^{2}+2\left\|\partial_{t} \widehat{\psi}(t)\right\|_{H^{1}(\Gamma)}^{2} \\
-2 \lambda\left\langle\partial_{t} \widehat{u}(t), \partial_{t} \widehat{\phi}(t)\right\rangle_{2} \leq 2 K_{1}\left\|\partial_{t} \widehat{\phi}(t)\right\|_{2}^{2}+2 K_{2}\left\|\partial_{t} \widehat{\psi}(t)\right\|_{2, \Gamma}^{2} \\
-\frac{2}{\varepsilon}\left[\left\langle\left(f_{1}^{\prime}\left(\bar{\phi}_{0}(t)+\varepsilon \widetilde{\phi}(\tau)+\varepsilon \widehat{\phi}(t)\right)-f_{1}^{\prime}\left(\bar{\phi}_{0}(t)\right)\right) \partial_{t} \bar{\phi}_{0}(t), \partial_{t} \widehat{\phi}(t)\right\rangle_{2}\right] \\
-2\left[\left\langle f_{1}^{\prime}\left(\bar{\phi}_{0}(t)+\varepsilon \widetilde{\phi}(\tau)+\varepsilon \widehat{\phi}(t)\right) \partial_{t} \widetilde{\phi}(\tau), \partial_{t} \widehat{\phi}(t)\right\rangle_{2}\right] \\
-\frac{2}{\varepsilon}\left[\left\langle\left(f_{2}^{\prime}\left(\bar{\psi}_{0}(t)+\varepsilon \widehat{\psi}(t)\right)-f_{2}^{\prime}\left(\bar{\psi}_{0}\right)\right) \partial_{t} \bar{\psi}_{0}(t), \partial_{t} \widehat{\psi}(t)\right\rangle_{2, \Gamma}\right] \\
+\left\|\partial_{t} \Delta \widetilde{\phi}(\tau)\right\|_{2}\left(1+\left\|\partial_{t} \widehat{\phi}(t)\right\|_{2}^{2}\right)+\left\|\partial_{t} \partial_{\mathbf{n}} \widetilde{\phi}(\tau)\right\|_{2, \Gamma}\left(1+\left\|\partial_{t} \widehat{\psi}(t)\right\|_{2, \Gamma}^{2}\right) .
\end{aligned}
$$

Our aim is to estimate all the remaining terms on the right-hand side of (3.19). Since $\partial_{t} \bar{\phi}_{0}(t), \partial_{t} \bar{\psi}_{0}(t)$ are bounded in $L^{2}(\Omega)$ and $L^{2}(\Gamma)$, respectively, it follows, with the help of estimates (2.9) and (2.18), that

$$
\begin{aligned}
-\frac{2}{\varepsilon} & {\left[\left\langle\left(f_{1}^{\prime}\left(\bar{\phi}_{0}(t)+\varepsilon \widetilde{\phi}(\tau)+\varepsilon \widehat{\phi}(t)\right)-f_{1}^{\prime}\left(\bar{\phi}_{0}(t)\right)\right) \partial_{t} \bar{\phi}_{0}(t), \partial_{t} \widehat{\phi}(t)\right\rangle_{2}\right] } \\
& \leq C_{3}\left\langle 1+\left|\widehat{\phi}(t) \| \partial_{t} \bar{\phi}_{0}(t)\right|,\left|\partial_{t} \widehat{\phi}(t)\right|\right\rangle_{2} \\
& \leq C_{4}\left(1+\left\|\partial_{t} \widehat{\phi}(t)\right\|_{2}^{2}+\|\widehat{\phi}(t)\|_{2}^{2}\right)+\frac{1}{2}\|\widehat{\phi}(t)\|_{H^{1}(\Omega)}^{2}+\left\|\partial_{t} \widehat{\phi}(t)\right\|_{H^{1}(\Omega)}^{2},
\end{aligned}
$$

where the constants $C_{3}, C_{4}$ depend on the norm of the initial data $\phi(0), \psi(0)$ and $u(0)$ in $H^{2}$. Similarly, we have

$$
\begin{aligned}
2\left[\left\langle f_{1}^{\prime}\left(\bar{\phi}_{0}(t)+\varepsilon \widetilde{\phi}(\tau)+\varepsilon \widehat{\phi}(t)\right) \partial_{t} \widetilde{\phi}(\tau), \partial_{t} \widehat{\phi}(t)\right\rangle_{2}\right] \\
\quad \leq C_{5}\left\|\partial_{t} \widetilde{\phi}(\tau)\right\|_{H^{2}(\Omega)}\left(1+\left\|\partial_{t} \widehat{\phi}(t)\right\|_{2}^{2}\right)
\end{aligned}
$$

and

$$
\begin{aligned}
& -\frac{2}{\varepsilon}\left[\left\langle\left(f_{2}^{\prime}\left(\bar{\psi}_{0}(t)+\varepsilon \widehat{\psi}(t)\right)-f_{2}^{\prime}\left(\bar{\psi}_{0}\right)\right) \partial_{t} \bar{\psi}_{0}(t), \partial_{t} \widehat{\psi}(t)\right\rangle_{2, \Gamma}\right] \\
& \quad \leq C_{6}\left(1+\left\|\partial_{t} \widehat{\psi}(t)\right\|_{2, \Gamma}^{2}+\|\widehat{\psi}(t)\|_{2, \Gamma}^{2}\right)+\frac{1}{2}\|\widehat{\psi}(t)\|_{H^{1}(\Gamma)}^{2}+\left\|\partial_{t} \widehat{\psi}(t)\right\|_{H^{1}(\Gamma)}^{2} .
\end{aligned}
$$

Multiplying now the first equation of (3.13) by $\partial_{t} \widehat{u}(t)$ and integrating over $\Omega$, we obtain

$$
\begin{aligned}
\partial_{t}\left(\|\nabla \widehat{u}(t)\|_{2}^{2}\right. & \left.+2\left\langle\partial_{t} \bar{u}_{0}(t), \widehat{u}(t)\right\rangle_{2}\right)+2 \varepsilon\left\|\partial_{t} \widehat{u}(t)\right\|_{2}^{2}+2 \lambda\left\langle\partial_{t} \widehat{u}(t), \partial_{t} \widehat{\phi}(t)\right\rangle_{2} \\
& =2\left\langle\partial_{t}^{2} \bar{u}_{0}(t), \widehat{u}(t)\right\rangle_{2} \leq\left\|\partial_{t}^{2} \bar{u}_{0}(t)\right\|_{\left(H^{1}(\Omega)\right)^{*}}\left(1+\|\widehat{u}(t)\|_{H^{1}(\Omega)}^{2}\right) .
\end{aligned}
$$


Then, combining (3.18)-(3.22) and adding the resulting inequality to (3.23), we get

$$
\frac{d}{d t} \Lambda(t) \leq C_{7}\left(1+\left\|\partial_{t}^{2} \bar{u}_{0}(t)\right\|_{\left(H^{1}(\Omega)\right)^{*}}^{2}+\left\|\partial_{t} \widetilde{\phi}(t / \varepsilon)\right\|_{H^{2}(\Omega)}+\left\|\partial_{t} \widetilde{u}(t / \varepsilon)\right\|_{2}\right) \Lambda(t),
$$

where we have set

$$
\begin{array}{r}
\Lambda(t):=\delta\left\|\partial_{t} \widehat{\phi}(t)\right\|_{2}^{2}+\left\|\partial_{t} \widehat{\psi}(t)\right\|_{2, \Gamma}^{2}+\|\widehat{\phi}(t)\|_{H^{1}(\Omega)}^{2}+\|\widehat{\psi}(t)\|_{H^{1}(\Gamma)}^{2} \\
+\|\nabla \widehat{u}(t)\|_{2}^{2}+2\left\langle\partial_{t} \bar{u}_{0}(t), \widehat{u}(t)\right\rangle_{2}+K .
\end{array}
$$

The positive constant $K$ will be chosen large enough such that $\Lambda(t) \geq 0$ for any $t \geq 0$, but we still need to estimate the term $\langle\widehat{u}(t)\rangle^{2}$. We integrate the first equation of (3.12) over $\Omega$ and, using the second equation, we find

$$
\begin{aligned}
\lambda\langle\widehat{u}(t)\rangle=\delta\langle & \left.\partial_{t} \widehat{\phi}(t)\right\rangle+\left\langle\partial_{t} \widehat{\psi}(t)\right\rangle_{\Gamma}+\beta\langle\widehat{\psi}(t)\rangle_{\Gamma} \\
& +\frac{1}{\varepsilon}\left\langle f_{1}\left(\bar{\phi}_{0}(t)+\varepsilon \widetilde{\phi}(\tau)+\varepsilon \widehat{\phi}(t)\right)-f_{1}\left(\bar{\phi}_{0}(t)\right)\right\rangle \\
& +\frac{1}{\varepsilon}\left\langle f_{2}\left(\bar{\psi}_{0}(t)+\varepsilon \widehat{\psi}(t)\right)-f_{2}\left(\bar{\psi}_{0}\right)\right\rangle_{\Gamma},
\end{aligned}
$$

where $\langle v\rangle_{\Gamma}$ stands for the average of $v$ over $\Gamma$.

Recalling Theorems 1 and 3 and the fact that the functions $\phi(t)=\bar{\phi}_{0}(t)+$ $\varepsilon \widetilde{\phi}(\tau)+\varepsilon \widehat{\phi}(t), \psi(t)=\bar{\psi}_{0}(t)+\varepsilon \widehat{\psi}(t)$ and $\bar{\phi}_{0}(t), \bar{\psi}_{0}(t)$ are uniformly bounded in $L^{\infty}$ with respect to $\varepsilon$, it follows from (3.25) that

$$
\lambda^{2}\langle\widehat{u}(t)\rangle^{2} \leq C_{8}\left(1+\left\|\partial_{t} \widehat{\phi}(t)\right\|_{2}^{2}+\left\|\partial_{t} \widehat{\psi}(t)\right\|_{2, \Gamma}^{2}+\|\widehat{\phi}(t)\|_{2}^{2}+\|\widehat{\psi}(t)\|_{2, \Gamma}^{2}\right) .
$$

Combining (3.24) with (3.26), choosing $K$ large enough and then applying Gronwall's inequality, taking into account the fact that (see (2.18))

$$
\int_{t}^{t+1}\left(1+\left\|\partial_{t}^{2} \bar{u}_{0}(s)\right\|_{\left(H^{1}(\Omega)\right)^{*}}^{2}+\left\|\partial_{t} \widetilde{\phi}(s / \varepsilon)\right\|_{H^{2}(\Omega)}+\left\|\partial_{t} \widetilde{u}(s / \varepsilon)\right\|_{2}\right) d s \leq C_{9},
$$

we find

$$
\begin{aligned}
\left\|\partial_{t} \widehat{\phi}(t)\right\|_{2}^{2}+\left\|\partial_{t} \widehat{\psi}(t)\right\|_{2, \Gamma}^{2}+\|\widehat{\phi}(t)\|_{H^{1}(\Omega)}^{2} & \\
& +\|\widehat{\psi}(t)\|_{H^{1}(\Gamma)}^{2}+\|\widehat{u}(t)\|_{H^{1}(\Omega)}^{2} \leq C_{10} e^{C_{11} t},
\end{aligned}
$$

where the constants $C_{10}$ and $C_{11}$ depend on $\|(\phi(0), \psi(0), u(0))\|_{\mathbb{V}_{2}}$. Finally, estimate (3.16) follows from (3.27) (cf. [18,19] for more details). This finishes the proof of the lemma.

On account of the asymptotic expansion (3.10), arguing as in [27], we deduce from estimates (3.14)-(3.16) that 
Corollary 7. Let $(\phi, \psi, u)$ be a solution to $\mathcal{P}_{\varepsilon}$ and $\left(\bar{\phi}_{0}, \bar{\psi}_{0}, \bar{u}_{0}\right)$ be a solution to $\mathcal{P}_{0}$ with initial data $(\phi(0), \psi(0))$. Then, there holds

$$
\begin{aligned}
\left\|\phi(t)-\bar{\phi}_{0}(t)\right\|_{H^{2}(\Omega)}+\left\|\psi(t)-\bar{\psi}_{0}(t)\right\|_{H^{2}(\Gamma)}+\left\|u(t)-\bar{u}_{0}(t)\right\|_{H^{2}(\Omega)} \\
\quad+\left\|\partial_{t} \phi(t)-\partial_{t} \bar{\phi}_{0}(t)\right\|_{2}+\left\|\partial_{t} \psi(t)-\partial_{t} \bar{\psi}_{0}(t)\right\|_{2, \Gamma} \\
\quad+\varepsilon\left\|\partial_{t} u(t)-\partial_{t} \bar{u}_{0}(t)\right\|_{2} \\
\leq C\left(\|u(0)-\mathcal{L}(\phi(0))\|_{H^{2}(\Omega)} e^{-\rho \frac{t}{\varepsilon}}+\varepsilon e^{L t}\right),
\end{aligned}
$$

where $\rho>0$ is a constant depending only on $\Omega, \Gamma$ and the positive constants $C, L$ depend on $\|(\phi(0), \psi(0), u(0))\|_{\mathbb{V}_{2}}$, but are independent of $\varepsilon$.

Corollary 8. The following estimates hold:

$$
\begin{aligned}
\left\|\partial_{t} u(t)\right\|_{2} \leq & Q\left(\|(\phi(0), \psi(0), u(0))\|_{\mathbb{V}_{2}}\right) \\
& \times\left[1+\frac{1}{\varepsilon}\|u(0)-\mathcal{L}(\phi(0))\|_{H^{2}(\Omega)} e^{-\rho \frac{t}{\varepsilon}}\right], \\
\|u(t)-\mathcal{L}(\phi(t))\|_{H^{2}(\Omega)} \leq & Q\left(\|(\phi(0), \psi(0), u(0))\|_{\mathbb{V}_{2}}\right) \\
& \times\left[\varepsilon+\|u(0)-\mathcal{L}(\phi(0))\|_{H^{2}(\Omega)} e^{-\rho \frac{t}{\varepsilon}}\right],
\end{aligned}
$$

where $\rho>0$ and the positive and monotone increasing function $Q$ are independent of $\varepsilon$.

We conclude this section with two estimates on the difference of two trajectories of $\left(S_{t}^{\varepsilon}, \mathbb{D}_{\varepsilon}^{M}\right)$ that are also necessary for the construction of exponential attractors in the next section. For the details of the proofs, the reader is referred to [18, Lemma 4.6] and [19, Lemma 17].

Lemma 9. Let $\left(\phi_{i}(t), \psi_{i}(t), u_{i}(t)\right)=S_{t}^{\varepsilon}\left(\phi_{0 i}, \psi_{0 i}, u_{0 i}\right), i=1,2$. Suppose that they belong to a bounded absorbing set in $\mathbb{V}_{2}$, of radius $R>0$. Then, the following estimates hold:

$$
\begin{aligned}
& \left\|\left(\phi_{1}-\phi_{2}\right)(t)\right\|_{H^{2}(\Omega)}^{2}+\left\|\left(\psi_{1}-\psi_{2}\right)(t)\right\|_{H^{2}(\Gamma)}^{2}+\left\|\left(u_{1}-u_{2}\right)(t)\right\|_{H^{2}(\Omega)}^{2} \\
& \quad \leq C e^{L t}\left(\left\|\phi_{01}-\phi_{02}\right\|_{H^{2}(\Omega)}^{2}+\left\|\psi_{01}-\psi_{02}\right\|_{H^{2}(\Gamma)}^{2}+\left\|u_{01}-u_{02}\right\|_{H^{2}(\Omega)}^{2}\right), \\
& \left\|\left(\phi_{1}-\phi_{2}\right)(t)\right\|_{H^{3}(\Omega)}^{2}+\left\|\left(\psi_{1}-\psi_{2}\right)(t)\right\|_{H^{3}(\Gamma)}^{2}+\left\|\left(u_{1}-u_{2}\right)(t)\right\|_{H^{3}(\Omega)}^{2} \\
& \leq C \frac{t+1}{t} e^{L t}\left(\left\|\phi_{01}-\phi_{02}\right\|_{H^{2}(\Omega)}^{2}+\| \psi_{01}\right. \\
& \left.\quad-\psi_{02}\left\|_{H^{2}(\Gamma)}^{2}+\right\| u_{01}-u_{02} \|_{H^{2}(\Omega)}^{2}\right),
\end{aligned}
$$

for all $t>0$, where the constants $C, L$ depend on $R$, but are independent of $\varepsilon$.

\section{Proof of Theorem 4}

The proof follows from the application of an abstract result (see [27, Prop.3.2] and references therein) which is reported below for the reader's convenience. 
Theorem 10. Let $\mathbb{B}_{\varepsilon} \subset \Phi, \varepsilon \in[0,1]$, be a family of closed and bounded subsets of the Banach space $\Phi$ and let $\Sigma^{\varepsilon}: \mathbb{B}_{\varepsilon} \rightarrow \mathbb{B}_{\varepsilon}$ be a family of maps which satisfy the following properties:

(1) There exists another Banach space $\Phi_{1}$, which is compactly embedded into $\Phi$, such that, for every $b_{1}^{\varepsilon}, b_{2}^{\varepsilon} \in \mathbb{B}_{\varepsilon}$, the following estimate holds:

$$
\left\|\Sigma^{\varepsilon} b_{1}^{\varepsilon}-\Sigma^{\varepsilon} b_{2}^{\varepsilon}\right\|_{\Phi_{1}} \leq C\left\|b_{1}^{\varepsilon}-b_{2}^{\varepsilon}\right\|_{\Phi},
$$

where the constant $C$ is independent of $\varepsilon$.

(2) There exist nonlinear "projectors" $\Pi_{\varepsilon}: \mathbb{B}_{\varepsilon} \rightarrow \mathbb{B}_{0}$ such that, for every $b^{\varepsilon} \in \mathbb{B}_{\varepsilon}$,

$$
\left\|\Sigma_{(k)}^{\varepsilon} b^{\varepsilon}-\Sigma_{(k)}^{0} \Pi_{\varepsilon} b^{\varepsilon}\right\|_{\Phi} \leq \varepsilon L^{k}, \quad \forall k \in \mathbb{N},
$$

where $\Sigma_{(k)}^{\varepsilon}$ denotes the $k$-th iteration of $\Sigma^{\varepsilon}$ and the constant $L$ is independent of $\varepsilon$.

Then, the discrete semiflows $\Sigma_{(k)}^{\varepsilon}$ possess a family of exponential attractors $\mathcal{M}_{\varepsilon}^{d}$ which satisfy the discrete versions of (2.23)-(2.26).

In our case, we set $\Phi=\mathbb{D}_{\varepsilon}^{M}$ and $\Phi_{1}=\mathbb{V}_{3}$. Note that $\mathbb{V}_{3}$ is compactly embedded into $\mathbb{D}_{\varepsilon}^{M}$. Then, recalling (2.9), we define the sets $\mathbb{B}_{\varepsilon} \subset \mathbb{D}_{\varepsilon}^{M}$, for every $\varepsilon \in(0,1]$, in the following way:

$$
\mathbb{B}_{\varepsilon}:=\left\{\left(\phi_{0}, \psi_{0}, u_{0}\right) \in \mathbb{D}_{\varepsilon}^{M}:\left\|\left(\phi_{0}, \psi_{0}, u_{0}\right)\right\|_{\mathbb{V}_{2}}^{2} \leq 2 Q_{1}\left(\left\|g_{1}\right\|_{2}^{2}+\left\|g_{1}\right\|_{2, \Gamma}^{2}\right)\right\} .
$$

Similarly, on account of (2.18), we set

$$
\begin{aligned}
\mathbb{B}_{0}:=\left\{\left(\phi_{0}, \psi_{0}, u_{0}\right) \in \mathbb{L}^{M}:\left\|\left(\phi_{0}, \psi_{0}\right)\right\|_{H^{2}(\Omega) \times H^{2}(\Gamma)}^{2}\right. & \\
& \left.\leq 2 Q_{2}\left(\left\|g_{1}\right\|_{2}^{2}+\left\|g_{1}\right\|_{2, \Gamma}^{2}\right)\right\} .
\end{aligned}
$$

These sets are uniform (with respect to $\varepsilon$ ) bounded absorbing sets for the semiflows (2.10) and (2.19) thanks to Theorems 1-2 and Theorem 3, respectively. Thus, there exists a time $T \geq 1$, independent of $\varepsilon$, such that

$$
S_{T}^{\varepsilon}\left(\mathbb{B}_{\varepsilon}\right) \subseteq \mathbb{B}_{\varepsilon}, \quad \forall \varepsilon \in[0,1] .
$$

Setting $\Sigma^{\varepsilon}=S_{T}^{\varepsilon}$, we easily realize that these maps satisfy the smoothing property (4.1), thanks to estimate (3.32). Besides, following [27], we define the projections $\Pi_{\varepsilon}: \mathbb{B}_{\varepsilon} \rightarrow \mathbb{B}_{0}$ by setting

$$
\Pi_{\varepsilon}\left(\phi_{0}, \psi_{0}, u_{0}\right):=\left(\lambda \phi_{0}+\varepsilon u_{0}, \psi_{0}, \mathcal{L}\left(\lambda \phi_{0}+\varepsilon u_{0}\right)\right),
$$

which are well defined since $\left|\lambda\left\langle\phi_{0}\right\rangle+\varepsilon\left\langle u_{0}\right\rangle\right|=\left|I_{\varepsilon}\left(\phi_{0}, u_{0}\right)\right| \leq M$. Thus, estimate (4.2) follows from estimate (3.28) and the obvious estimate

$$
\left\|\Pi_{\varepsilon}\left(\phi_{0}, \psi_{0}, u_{0}\right)-\left(\phi_{0}, \psi_{0}, \mathcal{L}\left(\phi_{0}\right)\right)\right\|_{\mathbb{V}_{2}} \leq C \varepsilon,
$$

which holds for every $\left(\phi_{0}, \psi_{0}, u_{0}\right) \in \mathbb{B}_{\varepsilon}$, for some $C>0$ independent of $\varepsilon$. Hence, Theorem 10 yields a discrete family of exponential attractors $\mathcal{M}_{\varepsilon}^{d}$ for the discrete semiflows $\Sigma_{n T}^{\varepsilon}$ acting on the absorbing sets $\mathbb{B}_{\varepsilon}$. We now set

$$
\mathcal{M}_{\varepsilon}:=\cup_{t \in[T, T+1]} S_{t}^{\varepsilon} \mathcal{M}_{\varepsilon}^{d} .
$$


Then, thanks to (3.28) and (3.30)-(3.32), we can argue as in [27] and deduce that $\mathcal{M}_{\varepsilon}$ fulfills assumptions (2.23), (2.25)-(2.26). Finally, property (2.24) follows from (3.29)-(3.30) (see also [27, Lemma 3.3]) and (3.31)-(3.32).

\section{Global attractors revisited}

In [19], the authors also establish the existence of the global attractor $\mathcal{A}_{\varepsilon}$ for $\left(S_{t}^{\varepsilon}, \mathbb{D}_{\varepsilon}^{M}\right)$ and its upper semicontinuity at $\varepsilon=0$ (see [19, Thm. 4.3]). Here we wish to improve the latter result, by enlarging the phase space for the temperature $u$, according to the approach followed in [18].

Let us set $\mathbb{Z}_{q}:=H^{q}(\Omega) \times H^{q}(\Gamma) \times H^{q-1}(\Omega)$, for any $q \geq 1$. Then, for any $\varepsilon \in(0,1]$, we introduce the set

$$
\mathbb{X}_{\varepsilon}^{M}:=\left\{(\phi, \psi, u) \in H^{2}(\Omega) \times H^{2}(\Gamma) \times H^{1}(\Omega): \psi=\phi_{\mid \Gamma},\left|I_{\varepsilon}\right| \leq M\right\},
$$

where $M \geq$ is given. This set is a complete metric space with the metric induced by the norm in $\mathbb{Z}_{2}$. From [18], we know that the semiflow $S_{t}^{\varepsilon}$ can be defined on $\mathbb{X}_{\varepsilon}^{M}$. The existence of a bounded absorbing set has already been proven in [18, Lemma 4.3], but here we give a slightly different estimate which shows, in particular, the uniformity with respect to $\varepsilon$.

Lemma 11. Let assumptions (2.6)-(2.8) be satisfied. Then, for any $\left(\phi_{0}, \psi_{0}, u_{0}\right) \in$ $\mathbb{X}_{\varepsilon}^{M}$, the trajectory $(\phi(t), \psi(t), u(t))=S_{t}^{\varepsilon}\left(\phi_{0}, \psi_{0}, u_{0}\right)$ satisfies the estimate

$$
\begin{aligned}
& \|(\phi(t), \psi(t), u(t))\|_{\mathbb{Z}_{2}}^{2}+\left\|\partial_{t} \phi(t)\right\|_{2}^{2}+\left\|\partial_{t} \psi(t)\right\|_{2, \Gamma}^{2} \\
& \quad+\int_{t}^{t+1}\left(\left\|\partial_{t} \phi(s)\right\|_{H^{1}(\Omega)}^{2}+\left\|\partial_{t} \psi(s)\right\|_{H^{1}(\Gamma)}^{2}+\varepsilon\left\|\partial_{t} u(s)\right\|_{2}^{2}\right) d s \\
& \leq Q\left(\left\|\left(\phi_{0}, \psi_{0}, u_{0}\right)\right\|_{\mathbb{Z}_{2}}^{2}\right) e^{-\rho t}+Q\left(\left\|g_{1}\right\|_{2}^{2}+\left\|g_{2}\right\|_{2, \Gamma}^{2}\right),
\end{aligned}
$$

for some $\rho>0$ and some positive increasing function $Q$ which are both independent of $\varepsilon$.

Proof. We proceed formally (see [18] for details on a rigorous argument). Consider system (2.1). Then, take the inner product in $L^{2}(\Omega)$ of the first equation with $\partial_{t} \phi(t)$ and the inner product in $L^{2}(\Gamma)$ of the second equation with $\partial_{t} \psi(t)$, respectively. Adding these relations together with the one obtained by taking the inner product of the first equation of $(2.2)$ with $u(t)$, we deduce that

$$
\begin{aligned}
\frac{1}{2} \frac{d}{d t}[ & \left.\|\nabla \phi(t)\|_{2}^{2}+\alpha\left\|\nabla_{\Gamma} \psi(t)\right\|_{2, \Gamma}^{2}+\beta\|\psi(t)\|_{2, \Gamma}^{2}+\varepsilon\|u(t)\|_{2}^{2}\right] \\
& +\frac{1}{2} \frac{d}{d t}\left[2\left\langle F_{1}(\phi(t)), 1\right\rangle_{2}+2\left\langle F_{2}(\psi(t)), 1\right\rangle_{2, \Gamma}\right] \\
& +\|\nabla u(t)\|_{2}^{2}+\delta\left\|\partial_{t} \phi(t)\right\|_{2}^{2}+\left\|\partial_{t} \psi(t)\right\|_{2, \Gamma}^{2} \\
= & \left\langle\partial_{t} \phi(t), g_{1}\right\rangle_{2}+\left\langle\partial_{t} \psi(t), g_{2}\right\rangle_{2, \Gamma},
\end{aligned}
$$


where $F_{i}(w)=\int_{0}^{w} f_{i}(y) d y, i=1,2$. Let us now take the inner product in $L^{2}(\Omega)$ of the first equation and second equation of (3.2) with $2 \xi \phi(t)$, and $2 \xi \psi(t)$, respectively, for some $\xi>0$ to be fixed below. Combining the resulting relations with (5.3), we get, for some $0<\kappa<\xi$,

$$
\frac{d}{d t} E(t)+\kappa E(t)=\Lambda_{1}(t)
$$

where

$$
\begin{aligned}
E(t):= & \|\nabla \phi(t)\|_{2}^{2}+\alpha\left\|\nabla_{\Gamma} \psi(t)\right\|_{2, \Gamma}^{2}+\beta\|\psi(t)\|_{2, \Gamma}^{2} \\
& +2\left\langle F_{1}(\phi(t)), 1\right\rangle_{2}+2\left\langle F_{2}(\psi(t)), 1\right\rangle_{2, \Gamma} \\
& +\varepsilon\|u(t)\|_{2}^{2}+\xi\left[\delta\|\phi(t)\|_{2}^{2}+\|\psi(t)\|_{2, \Gamma}^{2}\right]+E_{0},
\end{aligned}
$$

for some $E_{0}>0$ such that $E(t)$ is nonnegative (cf. (2.7)) and

$$
\begin{aligned}
\Lambda_{1}(t):= & 2 \kappa\left\langle F_{1}(\phi(t))-f_{1}(\phi(t)) \phi(t), 1\right\rangle_{2} \\
& +2 \kappa\left\langle F_{2}(\psi(t))-f_{2}(\psi(t)) \psi(t), 1\right\rangle_{2, \Gamma} \\
& -(2 \xi-\kappa)\left(\|\nabla \phi(t)\|_{2}^{2}+\alpha\left\|\nabla_{\Gamma} \psi(t)\right\|_{2, \Gamma}^{2}+\beta\|\psi(t)\|_{2, \Gamma}^{2}\right) \\
& -2(\xi-\kappa)\left[\left\langle f_{1}(\phi(t)), \phi(t)\right\rangle_{2}+\left\langle f_{2}(\psi(t)), \psi(t)\right\rangle_{2, \Gamma}\right] \\
& -2\left(\delta\left\|\partial_{t} \phi(t)\right\|_{2}^{2}+\left\|\partial_{t} \psi(t)\right\|_{2, \Gamma}^{2}\right)+\left\langle\partial_{t} \phi(t), g_{1}\right\rangle_{2} \\
& +\left\langle\partial_{t} \psi(t), g_{2}\right\rangle_{2, \Gamma}-2\|\nabla u(t)\|_{2}^{2}+2 \xi \kappa\left[\delta\|\phi(t)\|_{2}^{2}+\|\psi(t)\|_{2, \Gamma}^{2}\right] \\
& +\kappa \varepsilon\|u(t)\|_{2}^{2}+2 \xi \lambda\langle u(t), \phi(t)\rangle_{2}+\kappa E_{0} .
\end{aligned}
$$

Observe now that

$$
2 \xi \lambda\langle u, \phi\rangle_{2}=2 \xi \lambda\langle u-\langle u\rangle, \phi\rangle_{2}+2 \xi|\Omega| I_{\varepsilon}\langle u\rangle-2 \xi \varepsilon|\Omega|\langle u\rangle^{2} .
$$

On the other hand, we know that there exists a positive constant $\widehat{C}$ such that, for all $v \in H^{1}(\Omega)$,

$$
\|v-\langle v\rangle\|_{2}^{2}=\|v\|_{2}^{2}-|\Omega|\langle v\rangle^{2} \leq \widehat{C}\|\nabla v\|_{2}^{2} .
$$

Let us rewrite $\Lambda_{1}$ in the following way:

$$
\begin{aligned}
\Lambda_{1}(t):= & 2 \kappa\left[\left\langle F_{1}(\phi(t))-f_{1}(\phi(t)) \phi(t), 1\right\rangle_{2}+\left\langle F_{2}(\psi(t))-f_{2}(\psi(t)) \psi(t), 1\right\rangle_{2, \Gamma}\right] \\
& -(2 \xi-\kappa)\left(\|\nabla \phi(t)\|_{2}^{2}+\alpha\left\|\nabla_{\Gamma} \psi(t)\right\|_{2, \Gamma}^{2}+\beta\|\psi(t)\|_{2, \Gamma}^{2}\right) \\
& -2(\xi-\kappa)\left[\left\langle f_{1}(\phi(t)), \phi(t)\right\rangle_{2}+\left\langle f_{2}(\psi(t)), \psi(t)\right\rangle_{2, \Gamma}\right] \\
& -2 \xi \varepsilon|\Omega|\langle u\rangle^{2}+\left\langle\partial_{t} \phi(t), g_{1}\right\rangle_{2}+\left\langle\partial_{t} \psi(t), g_{2}\right\rangle_{2, \Gamma} \\
- & 2\left(\delta\left\|\partial_{t} \phi(t)\right\|_{2}^{2}+\left\|\partial_{t} \psi(t)\right\|_{2, \Gamma}^{2}\right)-2\|\nabla u(t)\|_{2}^{2} \\
& +2 \xi|\Omega| I_{\varepsilon}\langle u\rangle+\kappa \varepsilon\|u(t)\|_{2}^{2}-2 \kappa \varepsilon|\Omega|\langle u\rangle^{2} \\
& +2 \xi \kappa\left[\delta\|\phi(t)\|_{2}^{2}+\|\psi(t)\|_{2, \Gamma}^{2}\right]+2 \xi \lambda\langle u-\langle u\rangle, \phi\rangle_{2} .
\end{aligned}
$$


Then, recall that, owing to (2.10), we have

$$
\begin{aligned}
C_{*}\left|f_{i}(y)\right|(1+|y|) & \leq 2 f_{i}(y) y+C_{f_{i}}, \\
F_{i}(y)-f_{i}(y) y & \leq C_{f_{i}}^{\prime}|y|^{2}+C_{f_{i}}^{\prime \prime},
\end{aligned}
$$

for any $y \in \mathbb{R}$ and $i=1,2$. Here $C_{f_{i}}, C_{*}, C_{f_{i}}^{\prime}$ and $C_{f_{i}}^{\prime \prime}$ are positive, sufficiently large constants that depend on $f_{i}$ only. Using these inequalities, we infer from (5.7) that

$$
\begin{aligned}
\Lambda_{1}(t) \leq & -\left(2 \xi-\kappa-\kappa\left(C_{f_{1}}^{\prime}+C_{f_{2}}^{\prime}\right)-2 \xi \kappa\right)\left[\|\phi(t)\|_{H^{1}(\Omega)}^{2}+\|\psi(t)\|_{H^{1}(\Gamma)}^{2}\right] \\
& -\left(\delta\left\|\partial_{t} \phi(t)\right\|_{2}^{2}+\left\|\partial_{t} \psi(t)\right\|_{2, \Gamma}^{2}\right)+C_{\delta}\left(1+\left\|g_{1}\right\|_{2}^{2}+\left\|g_{2}\right\|_{2, \Gamma}^{2}\right) \\
& -2(\xi-\kappa)\left[\left\langle f_{1}(\phi(t)), \phi(t)\right\rangle_{2}+\left\langle f_{2}(\psi(t)), \psi(t)\right\rangle_{2, \Gamma}\right] \\
& +2 \xi|\Omega| I_{\varepsilon}\langle u\rangle-2(\xi-\kappa) \varepsilon|\Omega|\langle u\rangle^{2} \\
& -2(1-\kappa \varepsilon \widehat{C})\|\nabla u(t)\|_{2}^{2}+\xi^{2} \lambda^{2} \widehat{C}\|\phi(t)\|_{2}^{2} .
\end{aligned}
$$

Let us now estimate the average $\langle u\rangle$. Integrating equations (2.1) over $\Omega$ and $\Gamma$ and adding the resulting identities, we obtain

$$
\begin{aligned}
\frac{d}{d t} \varepsilon\langle u(t)\rangle+\frac{\lambda^{2}}{\delta}\langle u(t)\rangle= & \frac{\lambda|\Gamma|}{\delta|\Omega|}\left\langle\partial_{t} \psi\right\rangle_{\Gamma}+\frac{\lambda \beta|\Gamma|}{\delta|\Omega|}\langle\psi\rangle_{\Gamma} \\
& +\frac{\lambda|\Gamma|}{\delta|\Omega|}\left\langle f_{2}(\psi)\right\rangle_{\Gamma}+\frac{1}{\delta}\left\langle f_{1}(\phi)\right\rangle_{\Omega} .
\end{aligned}
$$

We multiply (5.9) by

$$
\zeta:=\frac{2 \xi \delta|\Omega| I_{\varepsilon}}{\lambda^{2}-\kappa \delta \varepsilon},
$$

provided that $\kappa \delta \varepsilon<\lambda^{2}$, and we add the resulting relation to (5.4). Then, we derive the inequality

$$
\begin{aligned}
\frac{d}{d t}[E(t)+ & \zeta \varepsilon\langle u(t)\rangle]+\kappa[E(t)+\zeta \varepsilon\langle u(t)\rangle] \\
\leq & -\left(2 \xi-\kappa-\kappa\left(C_{f_{1}}^{\prime}+C_{f_{2}}^{\prime}\right)-2 \xi \kappa-\xi^{2} \lambda^{2} \widehat{C}\right)\left[\|\phi(t)\|_{H^{1}(\Omega)}^{2}+\|\psi(t)\|_{H^{1}(\Gamma)}^{2}\right] \\
& -\left(\delta\left\|\partial_{t} \phi(t)\right\|_{2}^{2}+\left\|\partial_{t} \psi(t)\right\|_{2, \Gamma}^{2}\right)+C_{\delta}\left(1+\left\|g_{1}\right\|_{2}^{2}+\left\|g_{2}\right\|_{2, \Gamma}^{2}\right) \\
& -2(\xi-\kappa)\left[\left\langle f_{1}(\phi(t)), \phi(t)\right\rangle_{2}+\left\langle f_{2}(\psi(t)), \psi(t)\right\rangle_{2, \Gamma}\right]-2(\xi-\kappa) \varepsilon|\Omega|\langle u\rangle^{2} \\
& -2(1-\kappa \varepsilon \widehat{C})\|\nabla u(t)\|_{2}^{2}+\frac{\lambda|\Gamma|}{\delta|\Omega|}\left\langle\partial_{t} \psi\right\rangle_{\Gamma}+\frac{\lambda \beta|\Gamma|}{\delta|\Omega|}\langle\psi\rangle_{\Gamma} \\
& +\frac{\lambda|\Gamma|}{\delta|\Omega|}\left\langle f_{2}(\psi)\right\rangle_{\Gamma}+\frac{1}{\delta}\left\langle f_{1}(\phi)\right\rangle_{\Omega} .
\end{aligned}
$$


Due to assumption (2.11), we have that, for any $\eta>0$, there exists $C_{\eta}>0$ such that (cf. $[27,(4.16)])$

$$
\begin{gathered}
\left|\left\langle f_{1}(\phi(t))\right\rangle\right| \leq\left\langle\left|f_{1}(\phi(t))\right|\right\rangle \leq \eta\left\langle f_{1}(\phi(t)), \phi(t)\right\rangle_{2}+C_{\eta}, \\
\left|\left\langle f_{2}(\psi(t))\right\rangle_{\Gamma}\right| \leq\left\langle\left|f_{2}(\psi(t))\right|\right\rangle_{\Gamma} \leq \eta\left\langle f_{2}(\psi(t)), \psi(t)\right\rangle_{2, \Gamma}+C_{\eta} .
\end{gathered}
$$

Thus, thanks to the above inequalities, we deduce that (cf. also [19])

$$
\begin{aligned}
\frac{d}{d t}[E(t) & +\zeta \varepsilon\langle u(t)\rangle]+\kappa[E(t)+\zeta \varepsilon\langle u(t)\rangle] \\
& +\kappa^{\prime}\left(\|\phi(t)\|_{H^{1}(\Omega)}^{2}+\|\psi(t)\|_{H^{1}(\Gamma)}^{2}+\delta\left\|\partial_{t} \phi(t)\right\|_{2}^{2}+\left\|\partial_{t} \psi(t)\right\|_{2, \Gamma}^{2}+\|\nabla u(t)\|_{2}^{2}\right) \\
\leq & C\left(1+\left\|g_{1}\right\|_{2}^{2}+\left\|g_{2}\right\|_{2, \Gamma}^{2}\right),
\end{aligned}
$$

where $\kappa, \kappa^{\prime}$ and $C$ are positive constants that are independent of $\varepsilon$. On the other hand, one can easily check that there exists a positive constant $C$ independent of $\varepsilon$ such that, for all $t \geq 0$,

$$
\Lambda_{2}(t) \leq C(E(t)+\zeta \varepsilon\langle u(t)\rangle)
$$

where

$$
\Lambda_{2}(t)=\|\phi(t)\|_{H^{1}(\Omega)}^{2}+\|\psi(t)\|_{H^{1}(\Gamma)}^{2}+\varepsilon\|u(t)\|_{2}^{2} .
$$

Applying Gronwall's inequality to (5.13) and taking (5.14) into account, we obtain

$$
\begin{aligned}
& \Lambda_{2}(t)+\int_{t}^{t+1}\left(\|\phi(s)\|_{H^{1}(\Omega)}^{2}+\|\psi(s)\|_{H^{1}(\Gamma)}^{2}+\delta\left\|\partial_{t} \phi(s)\right\|_{2}^{2}\right) d s \\
& +\int_{t}^{t+1}\left(\left\|\partial_{t} \psi(s)\right\|_{2, \Gamma}^{2}+\|\nabla u(s)\|_{2}^{2}\right) d s \leq C\left(E(0) e^{-\rho t}+1+\left\|g_{1}\right\|_{2}^{2}+\left\|g_{2}\right\|_{2, \Gamma}^{2}\right),
\end{aligned}
$$

for all $t \geq 0$, for some $\rho>0$ and $C>0$ independent of $t$ and $\varepsilon$.

Hence, using (5.15) and arguing as in [18, Lemma 4.3, (4.20)-(4.23)], we deduce that

$$
\begin{aligned}
\left\|\partial_{t} \phi(t)\right\|_{2}^{2} & +\left\|\partial_{t} \psi(t)\right\|_{2, \Gamma}^{2}+\|\nabla u(t)\|_{2}^{2} \\
& +\int_{t}^{t+1}\left(\left\|\partial_{t} \phi(s)\right\|_{H^{1}(\Omega)}^{2}+\left\|\partial_{t} \psi(s)\right\|_{H^{1}(\Gamma)}^{2}+\varepsilon\left\|\partial_{t} u(s)\right\|_{2}^{2}\right) d s \\
\leq & Q\left(\left\|\left(\phi_{0}, \psi_{0}, u_{0}\right)\right\|_{\mathbb{X}_{\varepsilon} M}^{2}\right) e^{-\rho t}+Q\left(\left\|g_{1}\right\|_{2}^{2}+\left\|g_{2}\right\|_{2, \Gamma}^{2}\right)
\end{aligned}
$$

and it follows from (2.1) that

$$
\lambda\langle u(t)\rangle \leq Q\left(\left\|\left(\phi_{0}, \psi_{0}, u_{0}\right)\right\|_{\mathbb{X}_{\varepsilon}^{M}}^{2}\right) e^{-\rho t}+Q\left(\left\|g_{1}\right\|_{2}^{2}+\left\|g_{2}\right\|_{2, \Gamma}^{2}\right),
$$

for some $\rho>0$ and some positive increasing function $Q$, both independent of $\varepsilon$. Finally, (5.2) is derived from (5.15)-(5.17), arguing as in the final part of the proof of $[18$, Lemma 4.3]. 
The next lemma shows the existence of a compact absorbing set in $\mathbb{X}_{\varepsilon}^{M}$, namely,

Lemma 12. Let assumptions (2.6)-(2.8) be satisfied. There exists a positive monotone nondecreasing function $Q$ (independent of $\varepsilon$ ) and, for any $R_{0}>0$, there exists $t_{0}=t_{0}\left(R_{0}\right)>0$ such that

$$
\|(\phi(t), \psi(t), u(t))\|_{\mathbb{Z}_{3}} \leq Q\left(R_{0}\right), \quad \forall t \geq t_{0},
$$

for any $\left(\phi_{0}, \psi_{0}, u_{0}\right) \in \mathcal{B}\left(R_{0}\right) \subset \mathbb{X}_{\varepsilon}^{M}$, where $\mathcal{B}\left(R_{0}\right)$ is a ball of radius $R_{0}$, centered at 0 .

Proof. Following a formal argument (cf. [18, Lemma 4.4]), we differentiate the first two equations of (2.1) and the first equation of (2.2) with respect to time. Then, we multiply the resulting equations by $\partial_{t}^{2} \phi(t), \partial_{t}^{2} \psi(t)$ and $\partial_{t} u(t)$, respectively. Integrating by parts and adding the resulting relations, we deduce that

$$
\begin{gathered}
\frac{1}{2} \frac{d}{d t}\left[\left\|\partial_{t} \phi(t)\right\|_{H^{1}(\Omega)}^{2}+\left\|\partial_{t} \psi(t)\right\|_{H^{1}(\Gamma)}^{2}+\varepsilon\left\|\partial_{t} u(t)\right\|_{2}^{2}\right] \\
+\left\|\partial_{t}^{2} \phi(t)\right\|_{2}^{2}+\left\|\partial_{t}^{2} \psi(t)\right\|_{2, \Gamma}^{2}+\left\|\nabla \partial_{t} u(t)\right\|_{2}^{2} \\
=-\int_{\Omega} f_{1}^{\prime}(\phi(t)) \partial_{t} \phi(t) \partial_{t}^{2} \phi(t) d x-\int_{\Gamma} f_{2}^{\prime}(\psi(t)) \partial_{t} \psi(t) \partial_{t}^{2} \psi(t) d S .
\end{gathered}
$$

Using Hölder's and Young's inequalities, we get

$$
\begin{aligned}
\frac{d}{d t}\left(\left\|\partial_{t} \phi(t)\right\|_{H^{1}(\Omega)}^{2}+\left\|\partial_{t} \psi(t)\right\|_{H^{1}(\Gamma)}^{2}+\varepsilon\left\|\partial_{t} u(t)\right\|_{2}^{2}\right) \\
+\left\|\partial_{t}^{2} \phi(t)\right\|_{2}^{2}+\left\|\partial_{t}^{2} \psi(t)\right\|_{2, \Gamma}^{2}+\left\|\nabla \partial_{t} u(t)\right\|_{2}^{2} \\
\leq C\left(\left\|f_{1}^{\prime}(\phi(t)) \partial_{t} \phi(t)\right\|_{2}^{2}+\left\|f_{2}^{\prime}(\psi(t)) \partial_{t} \psi(t)\right\|_{2, \Gamma}^{2}\right)
\end{aligned}
$$

for some $C>0$ that is independent of $\varepsilon$ and of the initial data. On account of the embedding $H^{2}(\Omega) \hookrightarrow C(\bar{\Omega})$, we can find a positive monotone increasing function $Q$, independent of $\varepsilon$, such that

$$
\begin{aligned}
\frac{d}{d t}\left(\left\|\partial_{t} \phi(t)\right\|_{H^{1}(\Omega)}^{2}+\left\|\partial_{t} \psi(t)\right\|_{H^{1}(\Gamma)}^{2}+\varepsilon\left\|\partial_{t} u(t)\right\|_{2}^{2}\right) \\
\quad+\left\|\partial_{t}^{2} \phi(t)\right\|_{2}^{2}+\left\|\partial_{t}^{2} \psi(t)\right\|_{2, \Gamma}^{2}+\left\|\nabla \partial_{t} u(t)\right\|_{2}^{2} \\
\leq Q\left(\|\phi(t)\|_{H^{2}(\Omega)}\right)\left\|\partial_{t} \phi(t)\right\|_{2}^{2}+Q\left(\|\psi(t)\|_{H^{2}(\Gamma)}\right)\left\|\partial_{t} \psi(t)\right\|_{2, \Gamma}^{2}
\end{aligned}
$$

Recalling (5.2), we can apply the uniform Gronwall lemma to (5.21). This yields

$$
\left\|\partial_{t} \phi(t)\right\|_{H^{1}(\Omega)}^{2}+\left\|\partial_{t} \psi(t)\right\|_{H^{1}(\Gamma)}^{2}+\varepsilon\left\|\partial_{t} u(t)\right\|_{2}^{2} \leq Q\left(R_{0}\right), \quad \forall t \geq t_{0},
$$

where $t_{0}=t_{0}\left(R_{0}\right)>0$ is independent of $\varepsilon$. We can now use (5.2) and (5.22) to derive (5.18), arguing as in the proof of [18, Lemma 4.4].

We also recall the continuous dependence estimate (see [18, (3.42)]). 
Lemma 13. Let assumptions (2.6)-(2.8) be satisfied. Consider two trajectories originating from $\mathbb{X}_{\varepsilon}^{M}$, namely $\left(\phi_{i}(t), \psi_{i}(t), u_{i}(t)\right)=S_{t}^{\varepsilon}\left(\psi_{0 i}, \phi_{0 i}, u_{0 i}\right), i=1,2$. Then, the following estimate holds:

$$
\begin{aligned}
& \left\|\left(\phi_{1}-\phi_{2}\right)(t)\right\|_{H^{1}(\Omega)}^{2}+\left\|\left(\psi_{1}-\psi_{2}\right)(t)\right\|_{H^{1}(\Gamma)}^{2}+\varepsilon\left\|\left(u_{1}-u_{2}\right)(t)\right\|_{2}^{2} \\
& \quad \leq C e^{L t}\left(\left\|\phi_{01}-\phi_{02}\right\|_{H^{1}(\Omega)}^{2}+\left\|\psi_{01}-\psi_{02}\right\|_{H^{1}(\Gamma)}^{2}+\varepsilon\left\|u_{01}-u_{02}\right\|_{2}^{2}\right),
\end{aligned}
$$

where $C$ and $L$ are independent of $\varepsilon$.

On account of (5.2), (5.18) and (5.23), we deduce (cf. [31]) the

Corollary 14. Let assumptions (2.6)-(2.8) be satisfied. Then, for each $\varepsilon \in(0,1]$, the dynamical system $\left(S_{t}^{\varepsilon}, \mathbb{X}_{\varepsilon}^{M}\right)$ has a connected global attractor $\mathcal{A}_{\varepsilon}$ that is bounded in $\mathbb{Z}_{3}$.

Remark 2. It is worth noting that, in order to prove the result of Corollary 15, the nonlinearities $f_{i}$ need only be in $C^{1}(\mathbb{R})$.

We also recall that, owing to Theorem 4 , the dynamical system $\left(S_{t}^{0}, \mathbb{L}^{M}\right)$ has the global connected attractor $\mathcal{A}_{0}$ that is bounded in $\mathbb{V}_{3} \subset \mathbb{Z}_{3}$. More precisely, recalling (2.22), this attractor is defined by

$$
\mathcal{A}_{0}=\left\{(\phi, \psi, u) \in \mathbb{L}_{0}^{M}:(\phi, \psi) \in \widehat{\mathcal{A}}_{0}, u=\mathcal{L}(\phi)\right\},
$$

where $\widehat{\mathcal{A}}_{0}$ is the global attractor, bounded in $H^{3}(\Omega) \times H^{3}(\Gamma)$, of the dynamical system $\left(\widehat{S}_{t}^{0}, \widehat{\mathbb{L}}^{M}\right)$ generated by the viscous Cahn-Hilliard equation with dynamic boundary conditions.

Using estimates (5.2) and (5.22), we can argue as in the proof of [19, Theorem 4.3] to get the

Theorem 15. Let assumptions (2.6)-(2.8) be satisfied. Then, the family $\left\{\mathcal{A}_{\varepsilon}\right\}_{\varepsilon \in[0,1]}$ is upper semicontinuous at $\varepsilon=0$, that is,

$$
\lim _{\varepsilon \rightarrow 0^{+}} \operatorname{dist}_{\mathbb{X}_{\varepsilon}^{M}}\left(\mathcal{A}_{\varepsilon}, \mathcal{A}_{0}\right)=0
$$

where dist $t_{\mathbb{X}_{\varepsilon}^{M}}$ denotes the Hausdorff semidistance in $\mathbb{X}_{\varepsilon}^{M}$.

\section{Acknowledgements}

The second author was partially supported by the Italian PRIN Research Project 2006 Problemi a frontiera libera, transizioni di fase e modelli di isteresi.

\section{References}

[1] S. Aizicovici, E. Feireisl, F. Issard-Roch, Long time convergence of solutions to a phase-field system, Math. Methods Appl. Sci. 24 (2001), 277-287.

[2] P. W. Bates, S. Zheng, Inertial manifolds and inertial sets for the phase-field equations, J. Dynam. Differential Equations 4 (1992), 375-397. 
[3] D. Brochet, X. Chen, D. Hilhorst, Finite dimensional exponential attractor for the phase-field model, Appl. Anal. 49 (1993), 197-212.

[4] D. Brochet, D. Hilhorst, Universal attractor and inertial sets for the phase-field model, Appl. Math. Lett. 4 (1991), 59-62.

[5] M. Brokate, J. Sprekels, Hysteresis and Phase Transitions, Springer, New York, 1996.

[6] J. W. Cahn, J. E. Hilliard, Free energy of a nonuniform system. I. Interfacial energy, J. Chem. Phys. 28 (1958), 258-267.

[7] G. Caginalp, An analysis of a phase field model of a free boundary, Arch. Ration. Mech. Anal. 92 (1986), 205-245.

[8] R. Chill, E. Fašangová, J. Prüss, Convergence to steady states of solutions of the Cahn-Hilliard and Caginalp equations with dynamic boundary conditions, Math. Nachr. 13 (2006), 1448-1462.

[9] L. Cherfils, S. Gatti, A. Miranville, Existence of global solutions to the Caginalp phase-field system with dynamic boundary conditions and singular potentials, J. Math. Anal. Appl. 343 (2008), 557-566.

[10] L. Cherfils, A. Miranville, Some remarks on the asymptotic behavior of the Caginalp system with singular potentials, Adv. Math. Sci. Appl. 16 (2007), 107-129.

[11] L. Cherfils, A. Miranville, On the Caginalp system with dynamic boundary conditions and singular potentials, Appl. Math., to appear.

[12] A. Damlamian, N. Kenmochi, N. Sato, Subdifferential operator approach to a class of nonlinear systems for Stefan problems with phase relaxation, Nonlinear Anal. 23 (1994), 115-142.

[13] C. Dupaix, A singularly perturbed phase field model with a logarithmic nonlinearity: upper semicontinuity of the attractor, Nonlinear Anal. 41 (2000), 725-744.

[14] C. Dupaix, D. Hilhorst, A singularly perturbed phase field problem: upper-semicontinuity of the attractor, in "Nonlinear analysis and applications (Warsaw, 1994)", GAKUTO Internat. Ser. Math. Sci. Appl. 7, 101-112, Gakkōtosho, Tokyo, 1996.

[15] C. Dupaix, D. Hilhorst, I. N. Kostin, The viscous Cahn-Hilliard equations as a limit of the phase field model: lower semicontinuity of the attractor, J. Dynam. Differential Equations 11 (1999), 333-353.

[16] C. Dupaix, D. Hilhorst, Ph. Laurençot, Upper semicontinuity of the attractor for a singularly perturbed phase field model, Adv. Math. Sci. Appl. 8 (1998), 115-143.

[17] C. M. Elliott, S. Zheng, Global existence and stability of solutions to the phase-field equations, in "Free boundary problems", Internat. Ser. Numer. Math. 95, 46-58, Birkhäuser Verlag, Basel, 1990.

[18] C. G. Gal, M. Grasselli, The nonisothermal Allen-Cahn equation with dynamic boundary conditions, Discrete Contin. Dyn. Syst. 22 (2008), 1009-1040.

[19] S. Gatti, A. Miranville, Asymptotic behavior of a phase-field system with dynamic boundary conditions, in "Differential Equations: Inverse and Direct Problems" (A. Favini, A. Lorenzi, Eds.), Ser. Lect. Notes Pure Appl. Math. 251, 149-170, Chapman \& Hall/CRC, Boca Raton, 2006.

[20] M. Grasselli, H. Petzeltová, G. Schimperna, Long time behavior of solutions to the Caginalp system with singular potential, Z. Anal. Anwendungen 25 (2006), 51-72. 
[21] A. Jiménez-Casas, A. Rodríguez-Bernal, Asymptotic behaviour for a phase field model in higher order Sobolev spaces, Rev. Mat. Complut. 15 (2002), 213-248.

[22] V.K. Kalantarov, On the minimal global attractor of a system of phase field equations (Russian), Zap. Nauchn. Sem. Leningrad. Otdel. Mat. Inst. Steklov (LOMI) 188 (1991), Kraev. Zadachi Mat. Fiz. i Smezh. Voprosy Teor. Funktsii. 22, 70-86, 186 [translation in J. Math. Sci. 70 (1994), no. 3, 1767-1777].

[23] O. V. Kapustyan, An attractor of a semiflow generated by a system of phase-field equations without uniqueness of the solution (Ukrainian), Ukraïn. Mat. Zh. 51 (1999), 1006-1009 [Translation in Ukrainian Math. J. 51 (1999), no.7, 1135-1139 (2000)].

[24] Ph. Laurençot, Degenerate Cahn-Hilliard equation as limit of the phase-field equation with non-constant thermal conductivity, in "Free boundary problems, theory and applications (Zakopane, 1995)", Pitman Res. Notes Math. Ser. 363, 135-144, Longman, Harlow, 1996.

[25] G. B. McFadden, Phase-field models of solidification, Contemp. Math. 306 (2002), 107-145.

[26] A. Miranville, S. Zelik, Exponential attractors for the Cahn-Hilliard equation with dynamic boundary conditions, Math. Models Appl. Sci. 28 (2005), 709-735.

[27] A. Miranville, S. Zelik, Robust exponential attractors for singularly perturbed phasefield type equations, Electron. J. Differential Equations 63 (2002), 1-28.

[28] A. Novick-Cohen, The Cahn-Hilliard equation: Mathematical and modeling perspectives, Adv. Math. Sci. Appl. 8 (1998), 965-985.

[29] A. Novick-Cohen, On the viscous Cahn-Hilliard equation, in "Material instabilities in continuum mechanics (Edinburgh, 1985-1986)", Oxford Sci. Publ., 329-342, Oxford Univ. Press, New York, 1988.

[30] J. Prüss, R. Racke, S. Zheng, Maximal regularity and asymptotic behavior of solutions for the Cahn-Hilliard equation with dynamic boundary conditions, Ann. Mat. Pura Appl. (4) 185 (2006), 627-648.

[31] V. Pata, S. Zelik, Global attractors for semigroups of closed operators, Comm. Pure Appl. Anal. 6 (2007), 481-486.

[32] R. Racke, S. Zheng, The Cahn-Hilliard equation with dynamical boundary conditions, Adv. Differential Equations 8 (2003), 83-110.

[33] R. Rossi, Asymptotic analysis of the Caginalp phase-field model for two vanishing time relaxation parameters, Adv. Math. Sci. Appl. 13 (2003), 249-271.

[34] N. Sato, T. Aiki, Phase field equations with constraints under nonlinear dynamic boundary conditions, Commun. Appl. Anal. 5 (2001), 215-234.

[35] G. Schimperna, Abstract approach to evolution equations of phase field type and applications, J. Differential Equations 164 (2000), 395-430.

[36] B.E. E. Stoth, The Cahn-Hilliard equation as degenerate limit of the phase-field equations, Quart. Appl. Math. 53 (1995), 695-700.

[37] M. I. Vishik, L. A. Lyusternik, Regular degeneration and boundary layer for linear differential equations with small parameter, Uspekhi Mat. Nauk. 2 (1957), 3-122.

[38] H. Wu, S. Zheng, Convergence to equilibrium for the Cahn-Hilliard equation with dynamic boundary conditions, J. Differential Equations 204 (2004), 511-531. 
[39] Z. Zhang, Asymptotic behavior of solutions to the phase-field equations with Neumann boundary conditions, Commun. Pure Appl. Anal. 4 (2005), 683-693.

Ciprian G. Gal

Department of Mathematical Sciences

University of Missouri

Columbia, MO 65211

USA

e-mail: ciprian@math.missouri.edu

Maurizio Grasselli

Dipartimento di Matematica "F. Brioschi"

Politecnico di Milano

I-20133 Milano

Italy

e-mail: maurizio.grasselli@polimi.it

Alain Miranville

Laboratoire de Mathèmatiques et Applications

Université de Poitiers

F-86962 Chasseneuil Futuroscope Cedex

France

e-mail: Alain.Miranville@math.univ-poitiers.fr

Received: 11 July 2007.

Accepted: 18 February 2008. 